\title{
Distribution of Arrivals
}

Nirmala Kasturi

Abstract: Many different distributions have been discussed by a number of authors. It has been observed that there is still more scope for density functions on arrivals which take significant role in lifetime statistical analysis. This paper proposes one probability density function for selected random variable.

Key Words: Random variable, continuous probability distribution, arrival rate, density function, arrivals, integrability.

\section{INTRODUCTION}

We have seen the random variables "Time for the first arrival", "time for the $\mathrm{r}^{\text {th }}$ arrival", etc. Now the random variable of interest is instead of asking "In fixed time interval, how many arrivals take place?", "We ask in a particular interval how likely there are successive arrivals". This random variable is continuous if it holds up a p.d.f. $f(x)$ which is continuous over the time axis with $f(x)$ greater than or equal to zero and $\int_{-\infty}^{\infty} f(x) d x=1$

For $\left.\begin{array}{rl}n & =0 \\ n & =1\end{array}\right\}$ Cases are absurd since we consider the two consecutive arrivals in the time interval.

We consider $n=2$.

Case - 1: $\mathrm{n}=\mathbf{2}$

Here we propose

$$
\begin{aligned}
& f(x)=\lambda[2 p(2, t)-P(1, t)]-\cdots(1) \\
& f(x)=\lambda\left[2 \frac{e^{-\lambda t}(\lambda t)^{2}}{2 !}-e^{-\lambda t} \lambda t\right]
\end{aligned}
$$

Here $f(x)$ is integrable and in general probability of arriving one customer in particular interval is greater than probability of arriving two customers in same time interval.

In equation (1) twice the first term is more than second term. Therefore equation (1) will give absolutely a positive number.

The arrival rate $\lambda$ is positive quantity.

$$
\therefore f(x) \geq 0 \forall x
$$

$$
\begin{aligned}
& \text { And } \int_{-\infty}^{\infty} f(x) d x=\int_{0}^{\infty} f(x) d x \\
& \text { Now } \int_{0}^{\infty} \lambda[2 P(2, t)-P(1, t)] d t \\
& \int_{0}^{\infty} \lambda\left[2 \frac{(\lambda t)^{2} e^{-\lambda t}}{2 !}-\frac{e^{-\lambda t}(\lambda t)^{1}}{1 !}\right] d t \\
& \int_{0}^{\infty}\left[2 \frac{(\lambda t)^{2} e^{-\lambda t}}{2 !} \lambda-\frac{e^{-\lambda t}(\lambda t)^{1}}{1 !} \lambda\right] d t \\
& =1 \\
& \therefore \int_{-\infty}^{\infty} f(x) d x=\int_{0}^{\infty} f(x) d x=1 \text { for } n=2 \\
& \begin{array}{l}
0.015 \\
0.005
\end{array}
\end{aligned}
$$

Figure - 1

Case - 2: When $n$ value is equals to three:

$$
f(x)=\lambda[2 P(3, t)-P(2, t)]
$$

Here $\mathrm{f}(\mathrm{x})$ is integrable and in general probability of arriving two customers in particular interval is greater than probability of arriving three customers in that time interval. Also twice the $\mathrm{P}(3, \mathrm{t})$ is more than second term in equation (2)

Therefore equation (2) is a positive quantity.

The arrival rate $\lambda$ is a positive value.

$\therefore f(x) \geq 0 \forall x$

$\therefore \lambda[2 P(3, x)-P(2, x)] \geq 0$

$$
\begin{aligned}
& \int_{0}^{\infty} \lambda[2 P(3, t)-P(2, t)] d t \\
& \int_{0}^{\infty} \lambda\left[2 \frac{(\lambda t)^{3} e^{-\lambda t}}{3 !}-\frac{e^{-\lambda t}(\lambda t)^{2}}{2 !}\right] d t
\end{aligned}
$$


$\int_{0}^{\infty}\left[2 \frac{(\lambda t)^{3} e^{-\lambda t}}{3 !} \lambda-\frac{e^{-\lambda t}(\lambda t)^{2}}{2 !} \lambda\right] d t$

$=1$

$f(x)=\lambda[2 P(3, x)-P(2, x)]$ is p.d.f. function when $\mathrm{n}=3$.

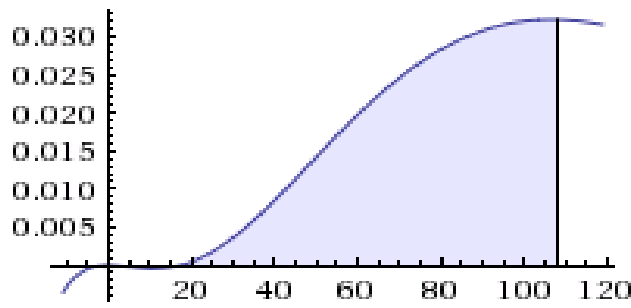

Figure - 2

Case - 3: $n=4$

$$
\begin{aligned}
& f(x)=\lambda[2 P(4, x)-P(3, x)] \\
& =\lambda\left[2 \frac{e^{-\lambda x}(\lambda x)^{4}}{4 !}-\frac{e^{-\lambda x}(\lambda x)^{3}}{3 !}\right]
\end{aligned}
$$

Here $\mathrm{f}(\mathrm{x})$ is integrable and since the probability of arriving four persons to system $\mathrm{P}(4, \mathrm{t})$ is less than probability of arriving 3 persons $P(3, t)$ to that system in the same time ' $t$ ' . Also twice $\mathrm{P}(4, \mathrm{t})$ is more than second term in equation (3).

Therefore equation (3) is absolutely positive value.

The arrival rate $\lambda$ is positive quantity.

$\therefore \lambda[2 P(4, t)-P(3, t)] \geq 0$

$\int_{0}^{\infty} \lambda[2 P(4, t)-P(3, t)] d t$

$\int_{0}^{\infty}\left[2 \frac{(\lambda t)^{4} e^{-\lambda t}}{4 !} \lambda-\frac{e^{-\lambda t}(\lambda t)^{3}}{3 !} \lambda\right] d t$

2(1) $-1=1$

$f(x)=\lambda[2 P(4, x)-P(3, x)]$ is p.d.f. function when $\mathrm{n}$ $=4$.

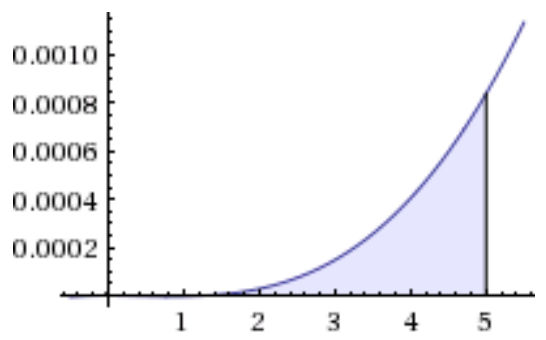

Figure -3

When it comes to the finite interval, we need to multiply the normalizer to get the area under the curve equals to 1 .

In general, we define

$f(x)=\left\{\begin{array}{l}\lambda[2 \Im(P(n, x)-P(n-1, x))] \text { when } x \geq 0 \\ 0 \text { otherwise }\end{array}\right.$

With $\mathfrak{I}$ is the normalizing constant.

\section{CONCLUSION}

In this paper we specified for the random variable for the successive arrivals, the probability density function,

$f(x)=\left\{\begin{array}{l}\lambda[2 \Im(P(n, x)-P(n-1, x))] \text { when } x \geq 0 \\ 0 \text { otherwise }\end{array}\right.$

\section{FUTURE WORK}

We can find CDF and all statistical properties of the above distribution.

\section{REFERENCES}

1. Daisuke Nakezato, Demitriics Bertsimas The distributional Little's law and its applications, (with D.Nakazato), Operations Research, 43, 2, 298 -310, MIT, 1995.

2. Demitriics BertsimasAn analytic approach to a general class of G/G/s queuing systems, Operations Research, 38, 1, 139-155, MIT, 1990

3. Hamdy Taha: Operations research An Introduction, seventh edition, pearson education.

4. James M Thomson and Donald Gross; Fundamentals of Queueing theory, Third addition, 2002: Wiley series.

5. Janus Sztrik, Queueing theory and its applications; A personal view by Proceedings of the 8th International conference on Applied Informatics, Eger, Hungary, Jan 27 - 30, 2010, Vol.1, PP.9-30.

6. Jeffrey Herrman: A survey of Queueing theory Applications in Healthcare, Samue Fomundam ISR Technical Report 2007-24.

7. J-P Cletch, D.M.Noctor, J.C.Manock, G.W.Lynott and F.E.Bader; Surface Mount Assembly failure statistics and failure free items AT\&T Bell laboratories, $44^{\text {th }}$ ECTC, Washington, D.C, May 1-4, 1994, PP.293-297.

8. Michael D. Peterson, Dimitris J. Bertsimas, Amedeo R. Odoni; MIT, Models and algorithms for Transient Queueing Congestion at Airports, Management science, volume 41, 1279-1295, 1995.

9. Noln Plumchitchom and Nick T. Thomopoulos; The Queueing theory of the Erlang Distributed Inter arrival and Service Time, Journal of Research in Engineering and Technology, Vol.3, No.4, October-December 2006

10. S.D. Sharma; Operations research, Fourteenth addition, 2003, Kedarnadh and Ramnadh and co. publishers. 
11. W. Weibull "A statistical distribution function of wide applicability" Journal of applied Mechanics, September 1951, pp 293-297.

12. R.Luus and Jammer; Estimation of parameters in 3 parameter Weibull probability distribution functions, $10^{\text {th }}$ international workshop on Chemical Engineering Mathematics, Budapet, Hungary, August 18-20 (2005).

13. Patric O'Conner, Reliability Engineering, $5^{\text {th }}$ Edition, 2012, Wiley Series.

14. William W. Hines, Douglas C. Montgomerry, David M.Goldsman, Connie M. Borrer, probability and Statistics in Engineering, Fourth Edition, Wiley Series.

15. Vijay K. Rohatgi, A.K.Md. Ehsanes Saleh, An Introduction to Probability And Statistics, Second Edition, Wiley Series.

16. Kishore S. Trivedi, Probability \& Statistics with Reliability, Queueing And Computer Science Applications, Second Edition, Wiley Series.

17. Ravindran, Phillips, Solberg, Operations Research principles and practice, Second Edition, Wiley series.

18. R.Sree parimala, S.Palaniammal Bulk Service Queueing Model With Services Single and Delayed Vacation, International Journal of Advances in Science and Technology(IJAST), Vol 2, Issue 2, June 2014

19. J. D. C. Little, "A Proof for the Queuing Formula: Operations Research, vol. 9(3), 1961, pp. 383-387, doi: 10.2307/167570.

20. M. Laguna and J. Marklund, Business Process Modeling, Simulation and Dsign, Pearson Prentice Hall, 2005.

21. Gamarnik, On the Undecidability of Computing Stationary Distributions and Large Deviation Rates for Constrained Random Walks, Mathematics of Operations Research, 2007, Vol. 32, 257265.

22. Gamarnik, Katz, On Deciding Stability of Multiclass Queueing Networks under Buffer Priority Scheduling Policies, Mathematics of Operations Research, 2009, Vol. 19, 2008-2037.

23. Ajeet Kumar Pandey, Srinivas Panchangam, Jessy George Smith, Safety analysis of Automatic Door operation for Metro Train : A case study. $9^{\text {th }}$ International conference on Heterogeneous Networking for quality, reliability Robustness, January 11-12-2013, Greater Noida, India.

24. Wallace Agiel, Christian Asave, Modeling and Analysis of Queueing Systems in Banks, International Journal of Scientific Technology, Volume 4, Issue 7, July 2015.

25. T.L. Pap and L. Leiszner, The use of Queueing Theory for planning automated analytical systems, Journal of Automated Chemistry, volume 9, No. 2 (April - June 1987), p.p 87-91.

26. X. Papaconstantinou, On the exact steady state solution of the Ek/C2/s queue, European Journal of Operations Research, 37(2), 272-287, 1988.

27. J Keilson, D, Nakazato, H, Transient and busy period analysis of the GI/G/1 queue as a Hilbert factorization problem, Journal of Applied Probability, 28, 873-885, 1991. 